\title{
Hemodialysis does not alter in vitro hepatic CYP3A4 and CYP2D6 metabolic activity in uremic serum
}

This article was published in the following Dove Press journal:

Clinical Pharmacology:Advances and Applications

9 December 2013

Number of times this article has been viewed

\author{
Brian S Decker ${ }^{1,2}$ \\ Kalisha D O'Neill ${ }^{1,2}$ \\ Mary A Chambers ${ }^{1,2}$ \\ James E Slaven ${ }^{3}$ \\ Zhangsheng $\mathrm{Yu}^{3}$ \\ David R Jones ${ }^{2,4}$ \\ Sharon M Moe ${ }^{1,2}$ \\ 'Division of Nephrology, ${ }^{2}$ Department \\ of Medicine, ${ }^{3}$ Department of \\ Biostatistics, ${ }^{4}$ Division of Clinical \\ Pharmacology, Department of \\ Medicine, School of Medicine, Indiana \\ University, Indianapolis, IN, USA
}

Correspondence: Brian S Decker Indiana University School of Medicine, 950 West Walnut St, R2-202, Indianapolis, IN 46254, USA

Tel + I 3172782865

Fax +l 3172748575

Email bsdecker@iu.edu

\begin{abstract}
There is a paucity of studies evaluating the change in liver metabolism in subjects receiving hemodialysis. The purpose of this study was to compare the effect of uremic toxins on hepatic cytochrome P450 (CYP)3A4 and CYP2D6 metabolism before and after a 4-hour hemodialysis session. Midazolam and dextromethorphan were incubated with uremic serum collected from subjects before and after the 4-hour hemodialysis session. Analysis and quantification of the 1'-OH-midazolam and 4-OH-midazolam and dextrorphan metabolites were performed by high-pressure liquid chromatography/mass spectrometry. Statistical analysis using the Student's $t$-test (paired) was used to compare the amount of metabolite formed. The mean amount of 1'-OH-midazolam, 4-OH-midazolam, and dextrorphan metabolites formed before and after hemodialysis did not significantly differ. There was no significant difference in CYP3A4 and CYP2D6 metabolic activity in uremic serum before and after hemodialysis.
\end{abstract}

Keywords: hemodialysis, uremia, CYP3A4, CYP2D6, metabolism

\section{Introduction}

Taking on average 12 medications to treat eight comorbid conditions, patients with end-stage renal disease (ESRD) are at tremendous risk for adverse drug events and drug interactions. ${ }^{1-3}$ A recent study estimates that nationwide nearly 22,000 adverse events occur annually in the ESRD patient population. ${ }^{2}$ Measures to prevent this in ESRD patients have focused on renal drug dosing algorithms that reduce the dose or frequency based on renal function with the assumption that reduced renal or dialysis clearance is the only mechanism by which drug metabolism is altered. However, this clinical approach to pharmacotherapy unfortunately neglects the contribution of hepatically metabolized medications that are typically given to ESRD patients at full doses.

In the liver, there are two sequential mechanisms important in drug metabolism, ie, uptake by transporters and metabolism by the cytochrome P450 (CYP) enzymes found in liver microsomes. Previous in vitro and animal studies have shown that hepatic CYP metabolism and drug transport is diminished by the uremic milieu found in ESRD. ${ }^{4-14}$ As a result, administering the usual full dose of a hepatically metabolized medication to an ESRD patient may place them at a similar risk for drug toxicity as administering the full dose of a renally eliminated medication. However, drug toxicity is not the only concern. Many medications are administered as prodrugs that require hepatic metabolism to achieve their active form. Thus, understanding liver metabolism in kidney disease and in the changing uremic milieu of hemodialysis is important. To date, there is a paucity of studies that have evaluated the change in liver metabolism in subjects receiving hemodialysis. 
In the present study, we expanded on these few scant human studies, using parallel methods to previous human studies to directly test the role of uremic toxins in blood before and after hemodialysis using human liver microsomes.

\section{Materials and methods}

\section{Study overview}

The in vitro metabolic activity of human CYP3A4 and CYP2D6 liver microsomes was evaluated given their central importance in the metabolism of the majority of medications. We used these microsomes to determine the effect of uremic serum on metabolism of midazolam and dextromethorphan. CYP3A4 is responsible for the majority of the hepatic metabolism of midazolam. ${ }^{15}$ Similarly, hepatic CYP2D6 metabolizes the majority of dextromethorphan with minor contributions from CYP3A4. ${ }^{16}$ The metabolites of interest for midazolam and dextromethorphan are 1'-OH-midazolam and 4-OHmidazolam and dextrorphan, respectively. ${ }^{15,16}$ Midazolam and dextromethorphan also share the characteristic that they do not require an active transport mechanism to gain entry to the enzymatic machinery of a hepatocyte. These medication probes are uniquely suited to evaluate the hepatic metabolism in microsomes because microsomes also lack transporters. As a result, any change in metabolic activity can be attributed to the CYP3A4 and CYP2D6 enzymes and not confounded by an effect on probe transporters. In this study, the extent of drug metabolism before and after a hemodialysis session was evaluated using human liver microsomes and the probes, midazolam and dextromethorphan, to test the hypothesis that acute clearance of uremic toxins will alter CYP3A4 and CYP2D6 metabolic activity.

\section{Study subjects}

Study approval was obtained from the Indiana University institutional review board. A total of 20 subjects with ESRD were recruited from the Indiana University hemodialysis unit. To meet the study criteria, subjects had to be 18 years or older, anuric, medically stable, receiving hemodialysis three times weekly, and have a stable $\mathrm{Kt} / \mathrm{V} \geq 1.2$ for the past 3 months. Subjects were excluded if they were pregnant, medically unstable, had liver disease, had missed more than one hemodialysis session in the 2 weeks prior to study entry, or were taking any CYP3A4 or CYP2D6 enzyme inducers or inhibitors.

\section{Materials}

Midazolam and dextromethorphan were obtained from Sigma-Aldrich (St Louis, MO, USA). The 1'-OH-midazolam, 4-OH-midazolam, and dextrorphan were purchased from
Toronto Research Chemicals Inc. (Ontario, Canada). The human liver microsomes pooled from 50 donors were obtained from Life Technologies (Carlsbad, CA, USA) and the recombinant liver microsomes were obtained from BD Biosciences (San Jose, CA, USA). Uremic serum was collected from 20 subjects before and after a single, approximately 4-hour hemodialysis session. The blood samples were drawn at the beginning just before the hemodialysis session (time zero) and 30 minutes after the conclusion of their hemodialysis session to allow equilibration of uremic toxins in the extracellular space. To confirm uremic solute clearance during hemodialysis, the blood samples were also analyzed for blood urea nitrogen concentrations. All blood samples were immediately processed for serum and stored at $-80^{\circ} \mathrm{C}$ for subsequent analysis. All other reagents and supplies were from standard commercial sources.

\section{Midazolam and dextromethorphan metabolism assays}

The procedure for the metabolic assays was conducted in a similar fashion for the human liver microsomes according to a previously reported technique. ${ }^{17}$ Human liver microsomes were used for the in vitro assays given their original human source and the expectation that the human liver microsomes would more closely replicate human hepatic metabolism. All assays were performed in quadruplicate. The metabolismincubation suspension consisted of microsomal protein, $100 \mathrm{mM}$ sodium phosphate buffer ( $\mathrm{pH} 7.4$ ) containing $5 \mathrm{mM}$ magnesium chloride, and $1 \mathrm{mM}$ of nicotinamide adenine dinucleotide phosphate. The amount of human microsomal protein in each incubation was $0.5 \mathrm{mg}$. The amounts of CYP3A4 (+b5) and CYP2D6 were 74 pmol and 42 pmol, respectively. The concentration of midazolam was $5 \mu \mathrm{M}$ and the concentration of dextromethorphan was $1 \mu \mathrm{M}$, both concentrations close to their Michaelis constant, Km. Given that the human liver microsomes contain the entire cassette of CYP enzymes, midazolam and dextromethorphan were incubated together. The incubation mixture was incubated for 6 minutes and terminated by the addition of $1 \mathrm{~mL}$ of methanol. After reaction termination, triazolam and codeine were added as internal standards and the samples were stored at $-80^{\circ} \mathrm{C}$ until analysis by liquid chromatography-tandem mass spectrometry (HPLC-MS/MS, Thermo Quantum Ultra, Thermo Fisher Scientific, Waltham, MA, USA).

\section{Blood urea nitrogen assays}

To confirm uremic solute clearance during hemodialysis, the blood samples before and after hemodialysis of the 20 ESRD 
subjects were analyzed for blood urea nitrogen concentrations using the QuantiChrom ${ }^{\mathrm{TM}}$ Urea Assay Kit (BioAssay Systems, Hayward, CA, USA).

\section{HPLC-MS/MS determinations of midazolam and dextromethorphan metabolites}

Dextrorphan, 1'-OH-midazolam, and 4-OH-midazolam were quantified by internal standardization (triazolam for the midazolam metabolites and codeine for dextrorphan) and HPLC-MS/MS. The metabolites were extracted from the incubation precipitant using $\mathrm{NaOH} /$ glycine $(\mathrm{pH} 11.3)$ and ethyl acetate. The ethyl acetate was evaporated to dryness, reconstituted with mobile phase, and injected into the HPLC-MS/MS. All metabolites and internal standards were resolved using a linear gradient of methanol:5 mM ammonium acetate ( $\mathrm{pH} 4.0)$ and an Eclipse XDB C18 $150 \mathrm{~mm} \times 4.6 \mathrm{~mm} 5 \mu \mathrm{m}$ column (Agilent Technologies, Santa Barbara, CA, USA). The Q1/Q3 for each analyte was dextrorphan (258/157), codeine (300/166), 4-OH (342/297), $1^{\prime}-\mathrm{OH}(342 / 324)$, and triazolam (343/308).

\section{Analysis of data and statistics}

The metabolite data represent the mean of quadruplicate assays for the quantity of 1'-OH-midazolam, 4-OHmidazolam, and dextrorphan formed from the human liver microsomes. The Student's $t$-test (paired) was utilized to compare the quantity of midazolam and dextromethorphan metabolites formed from the human liver microsomes before and after a hemodialysis session.

\section{Results}

Thirteen men and seven women with ESRD receiving three times weekly hemodialysis completed phase 2 of this study. The demographic and comorbid disease data for these subjects is found in Table 1. We compared the mean amount of 1'-OH-midazolam, 4-OH-midazolam, and dextrorphan metabolites formed just prior to dialysis at time zero and 30 minutes after the completion of the subject's hemodialysis session to test the hypothesis that acute uremic toxin removal with dialysis would alter metabolism. No significant difference in the amount of these metabolites was found after a 4-hour hemodialysis session. These results are shown Figures 1-3. In contrast and as expected, the blood urea nitrogen concentrations of the study subjects (Figure 4) demonstrated the expected decreases after hemodialysis, confirming uremic solute clearance. Statistical analysis using the Student's $t$-test did not show a significant difference
Table I Demographic and comorbid disease of study subjects

\begin{tabular}{lllll}
\hline Subject & Age & Sex & Ethnicity & Comorbid disease \\
\hline I & 63 & F & African-American & HTN, DM, PVD \\
2 & 45 & M & Caucasian & HTN, CAD \\
3 & 46 & M & African-American & HTN \\
4 & 50 & M & African-American & HTN \\
5 & 50 & F & African-American & HTN \\
6 & 45 & M & African-American & CAD, HTN \\
7 & 37 & M & African-American & HTN, CAD \\
8 & 65 & M & African-American & HTN, DM, PVD \\
9 & 83 & F & African-American & HTN, DM, PVD \\
10 & 53 & M & African-American & HTN \\
11 & 68 & F & African-American & HTN, DM, PVD \\
12 & 51 & M & African-American & HTN, CAD \\
13 & 40 & M & African-American & HTN, DM \\
14 & 57 & M & African-American & HTN, DM \\
15 & 60 & F & African-American & HTN, DM \\
16 & 42 & F & African-American & HTN \\
17 & 44 & M & African-American & HTN, CAD \\
18 & 29 & M & African-American & HTN, DM \\
19 & 55 & M & African-American & HTN, seizure \\
& & & & disorder \\
20 & 61 & F & African-American & HTN, DM, CAD \\
\hline
\end{tabular}

Abbreviations: HTN, hypertension; DM, diabetes mellitus; CAD, coronary artery disease; PVD, peripheral vascular disease.

between the midazolam or dextromethorphan metabolites formed prior to hemodialysis as compared with 30 minutes after the end of hemodialysis (Table 2).

\section{Discussion}

This ex vivo study is the first to evaluate the effects of uremic sera from hemodialysis patients on the metabolism of midazolam and dextromethorphan using human liver microsomes. We did not find a significant difference in the mean amount of midazolam or dextromethorphan metabolites formed in the serum of uremic patients before and after hemodialysis. Our findings are in contrast with the previous rat studies of uremia that incubated rat liver microsomes with normal and uremic serum and showed a decrement in CYP metabolic activity in uremic serum. ${ }^{4-14}$ Moreover, our results diverged from the initial research by Nolin et al of ESRD patients that showed an acute improvement in CYP3A4 metabolic activity with hemodialysis. ${ }^{18}$ The reasons that our human data are not consistent with the earlier animal studies may first be due to the limitations in the animal chronic kidney disease model. The rats in these earlier animal studies of uremia received 5/6 nephrectomies, and therefore were not end-stage and not dialyzed as in our human subjects. Second, there are physiologic differences in the CYP enzymes of rats and humans. Rat and human liver microsomes, although they have largely similar nomenclature, actually have different 


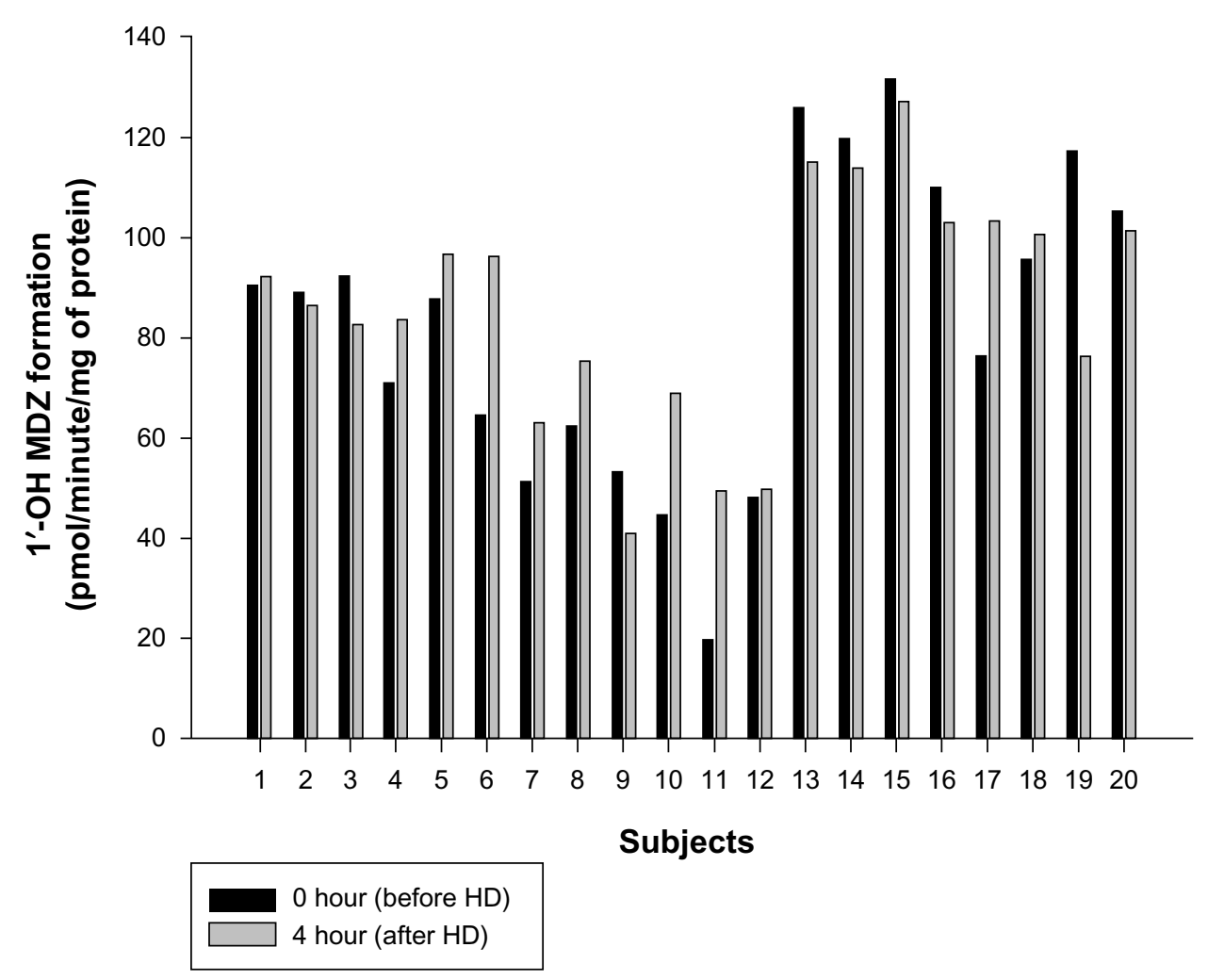

Figure I Formation of I'-OH-midazolam metabolite before and after hemodialysis. Abbreviations: $H D$, hemodialysis; MDZ, midazolam.

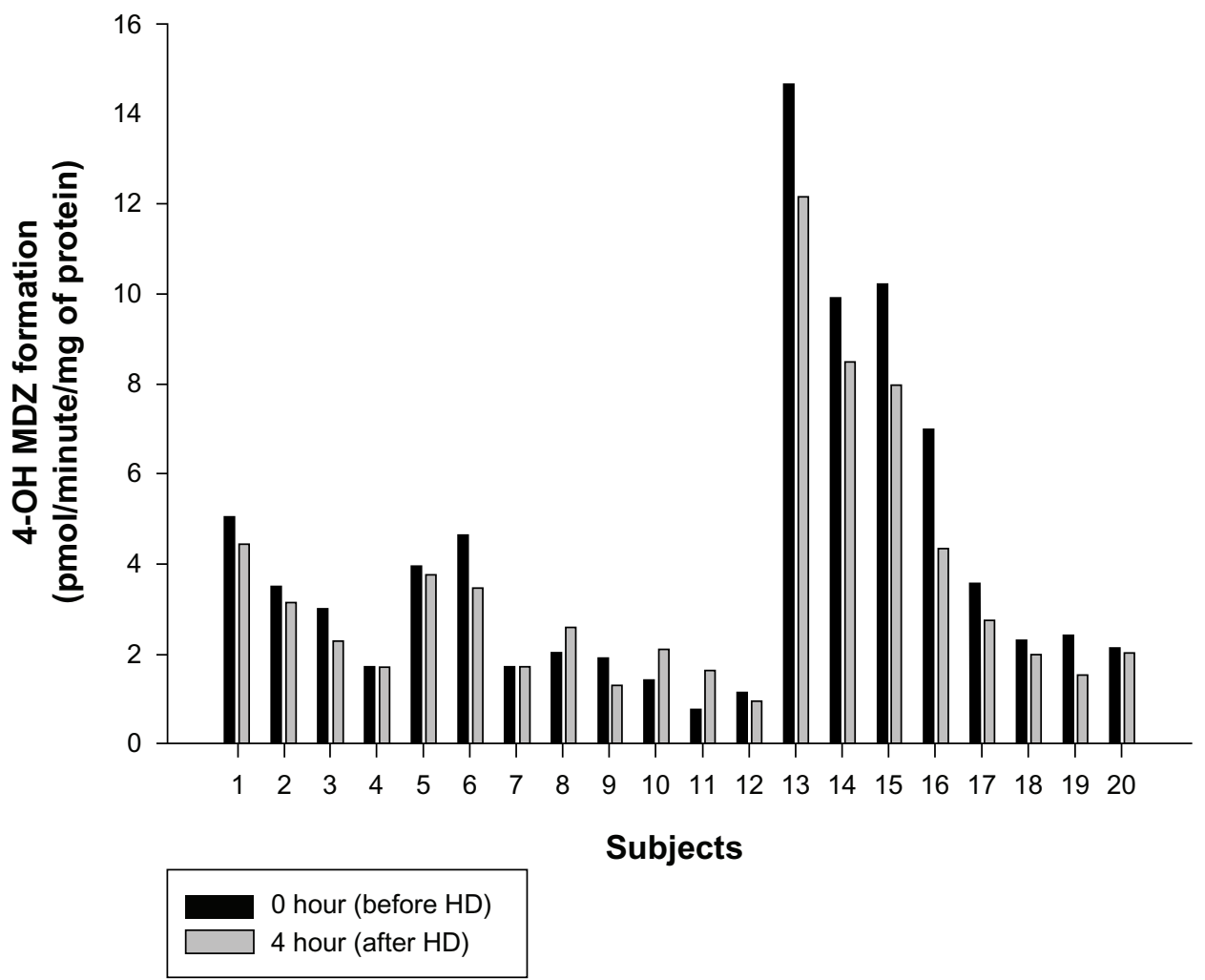

Figure 2 Formation of 4-OH-midazolam metabolite before and after hemodialysis. Abbreviations: $\mathrm{HD}$, hemodialysis; MDZ, midazolam. 


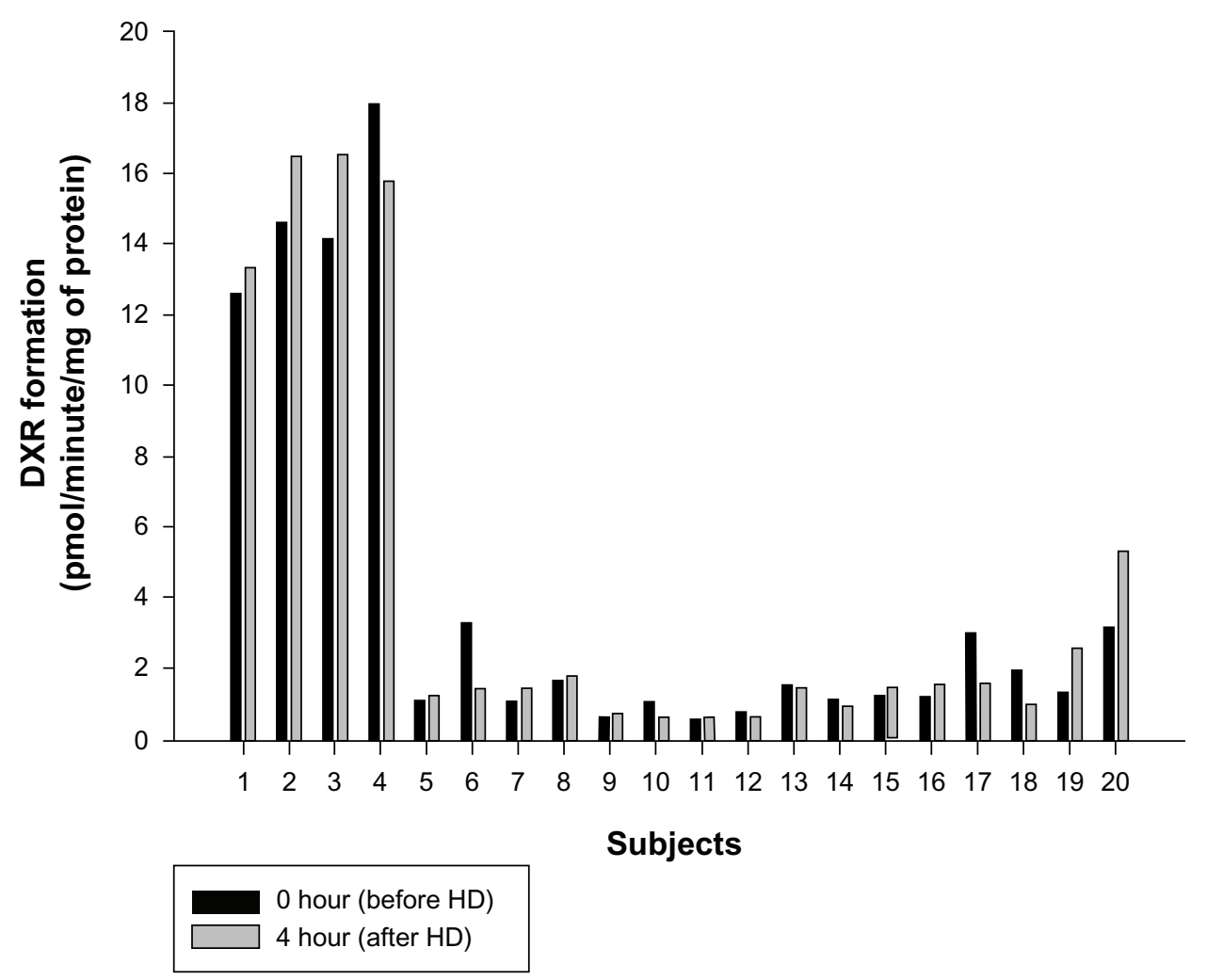

Figure 3 Formation of dextrorphan metabolite before and after hemodialysis. Abbreviations: DXR, dextrorphan; HD, hemodialysis.

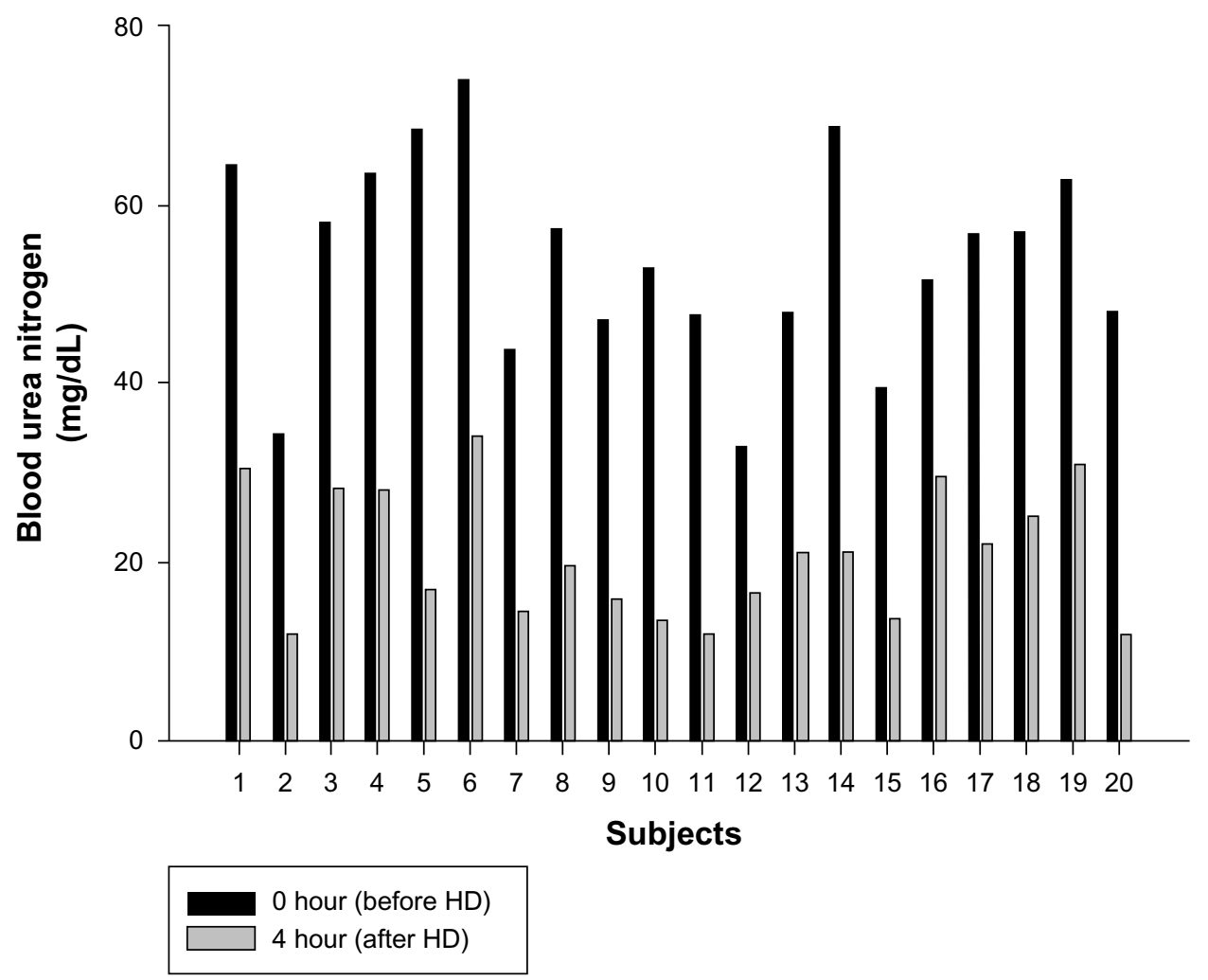

Figure 4 Blood urea nitrogen concentrations before and after hemodialysis. Abbreviation: HD, hemodialysis. 
Table 2 Comparison of mean metabolite formation before and after hemodialysis

\begin{tabular}{|c|c|c|c|}
\hline & $\begin{array}{l}\text { Metabolite formation with human } \\
\left.\text { liver microsomes (pmol* } \mathrm{min}^{-1 *} \mathrm{mgP}^{-1}\right) \\
\text { HD start, time } 0\end{array}$ & $\begin{array}{l}\text { Metabolite formation with human liver } \\
\left.\text { microsomes ( } \mathrm{pmol}^{*} \mathrm{~min}^{-1 *} \mathrm{mgP}^{-1}\right) \\
30 \text { minutes after HD session completed }\end{array}$ & $P$-value \\
\hline I'-OH-midazolam & $1.37(0.50)$ & $\mathrm{I} .42(0.39)$ & 0.755 \\
\hline 4-OH-midazolam & $0.86(0.46)$ & $0.78(0.43)$ & 0.620 \\
\hline Dextrorphan & $\mathrm{I} .48(0.73)$ & $1.56(1.08)$ & 0.799 \\
\hline
\end{tabular}

Notes: Values are shown as the mean (standard deviation); statistical significance, $P<0.05$.

Abbreviation: HD, hemodialysis.

substrate affinities. ${ }^{19,20}$ Chemical probes used for human CYP isoforms do not always have the same selectivity for the corresponding rat CYP isoforms. ${ }^{19,20}$ Third, hemodialysis is inherently limited in the magnitude of uremic toxin clearance it can provide. A 4-hour session of hemodialysis does not return an ESRD patient to a uremic milieu comparable with that of a normal subject without renal failure.

The departure of our findings from the initial human study of uremia and hemodialysis by Nolin et al ${ }^{18}$ also suggests that a second inhibitory pathway such as drug transport may be involved. Another human study conducted by Nolin et $\mathrm{a}^{21}$ using midazolam to evaluate CYP3A4 activity and fexofenadine to assess drug transport in patients with ESRD and normal renal function found no difference in CYP3A4 activity in the ESRD and normal renal function subjects. However, the investigators did find substantially reduced fexofenadine transport in the ESRD subjects, compared with the normal renal function controls. Since we used human liver microsomes which lack transporters, the absence of a difference in CYP3A4 and CYP2D6 metabolic activity before and after hemodialysis corroborates these results.

The primary strength of our study is the relatively large sample size of 20 subjects, greater than all of the previous studies of uremia in humans. ${ }^{18,21,22}$ In addition, we utilized human liver microsomes in our in vitro analysis, which permitted us to isolate the specific effect of uremic toxins on CYP450 enzyme activity without any potential confounding by hepatic transporters. Finally, because we used human liver microsomes and human uremic serum, we believe our in vitro methodology most closely replicates the true effect of uremia on human hepatic metabolism compared with the previous in vitro research in this area.

A potential limitation of our study is that we evaluated the acute effects of conventional, 4-hour hemodialysis on changes in CYP3A4 and CYP2D6 metabolic activity. It is possible that comparing different dialysis modalities (eg, daily versus three times weekly), or different stages of chronic kidney disease may have found a difference. Another potential limitation of this study is the ethnic homogeneity of the study population. Nearly all of the enrolled subjects were AfricanAmericans. This may limit the general applicability of our results, given the pharmacogenetic differences that may exist between ethnic groups.

In conclusion, this is the first ex vivo study to evaluate the acute effects of serum from hemodialysis patients on the metabolism of midazolam and dextromethorphan using human liver microsomes and human uremic serum. Our study demonstrated that acute removal of uremic toxins during hemodialysis does not alter CYP2D6 or CYP3A4 activity. Moreover, it provides additional corroborative evidence of the research by Nolin et a ${ }^{18}$ that uremia does not affect CYP2D6 and CYP3A4 activity. Given that collectively approximately $75 \%$ of drugs are metabolized by CYP3A4 and CYP2D6, this has important clinical implications. However, our work does leave open the possibility that hepatic transporters may be altered in uremia. This would require direct visualization of liver transport activity in order to determine the effect of uremia on hepatic transport. Such studies are ongoing in our laboratory.

\section{Acknowledgment}

Funding for this research was provided by a grant from the Indiana University Health Values Fund for Research and the National Institutes of Health (1K23KD084260-01A1).

\section{Author contributions}

All authors listed contributed sufficiently to the project to be included as authors.

\section{Disclosure}

The authors report no financial or other conflicts of interest in this work.

\section{References}

1. Kaplan B, Mason NA, Shimp LA, Ascione FJ. Chronic hemodialysis patients. Part I: Characterization and drug-related problems. Ann Pharmacother. 1994;28:316-319.

2. Manley HJ, Drayer DK, Muther RS. Medication-related problem type and appearance rate in ambulatory hemodialysis patients. BMC Nephrol. 2003;4:10 
3. Manley HJ, McClaran ML, Overbay DK, et al. Factors associated with medication-related problems in ambulatory hemodialysis patients. Am J Kidney Dis. 2003;41:386-393.

4. Leblond FA, Guevin C, Demers C, Pellerin I, Gascon-Barre M, Pichette V. Downregulation of hepatic cytochrome P450 in chronic renal failure. J Am Soc Nephrol. 2001;12:326-332.

5. Guevin C, Michaud J, Naud J, Leblond FA, Pichette V. Down-regulation of hepatic cytochrome p450 in chronic renal failure: role of uremic mediators. Br J Pharmacol. 2002;137:1039-1046.

6. Michaud J, Dube P, Naud J, et al. Effects of serum from patients with chronic renal failure on rat hepatic cytochrome P450. Br J Pharmacol. 2005;144:1067-1077.

7. Yu C, Ritter JK, Krieq RJ, Rege B, Karnes TH, Sarkar MA. Effect of chronic renal insufficiency on hepatic and renal UDPglucuronyltransferases in rats. Drug Metab Dispos. 2006;34:621-627.

8. Leblond FA, Giroux L, Villeneuve JP, Pichette V. Decreased in vivo metabolism of drugs in chronic renal failure. Drug Metab Dispos. 2000;28:1317-1320

9. Sun H, Frassetto L, Benet LZ. Effects of renal failure on drug transport and metabolism. Pharmacol Ther. 2006;109:1-11.

10. Nolin TD, Naud J, Leblond FA, Pichette V. Emerging evidence of the impact of kidney disease on drug metabolism and transport. Clin Pharmacol Ther. 2008;83:898-903.

11. Nolin TD, Frye RF, Matzke GR. Hepatic drug metabolism and transport in patients with kidney disease. Am J Kidney Dis. 2003;42: 906-925.

12. Dreisbach AW, Lertora JJ. Cytochrome P4502C9 activity in endstage renal disease. Clin Pharmacol Ther. 2003;73:475-477. Dreisbach AW, Lertora JJ. The effect of chronic renal failure on hepatic drug metabolism and drug disposition. Semin Dial. 2003;16:45-50.

13. Veau C, Leroy $\mathrm{C}$, Banide $\mathrm{H}$, et al. Effect of chronic renal failure on the expression and function of rat intestinal P-glycoprotein in drug excretion. Nephrol Dial Transplant. 2001;16:1607-1614.
14. Michaud J, Nolin TD, Naud J, et al. Effect of hemodialysis on hepatic cytochrome P450 functional expression. J Pharmacol Sci. 2008;108: 157-163.

15. Wandel C, Bocker R, Bohrer H, Browne A, Ruqheimer E, Martin E. Midazolam is metabolized by at least three different cytochrome P450 enzymes. Br J Anaesth. 1994;73:658-661.

16. Jacqz-Aigrain E, Funck-Brentano C, Crestiel T. CYP2D6- and CYP3A-dependent metabolism of dextromethorphan in humans. Pharmacogenetics. 1993;3:197-204.

17. Gorski JC, Jones DR, Wrighton SA, Hall SD. Characterization of dextromethorphan N-demethylation by human liver microsomes. Biochem Pharmacol. 1994;48:173-182.

18. Nolin TD, Appiah K, Kendrick SA, Le P, McMonagle E, Himmelfarb J. Hemodialysis acutely improves hepatic CYP3A4 metabolic activity. J Am Soc Nephrol. 2006;17:2363-2367.

19. Martignoni M, Geny MM, deKanter G, deKanter R. Species differences between mouse, rat, dog, monkey and human CYP-mediated drug metabolism, inhibition and induction. Expert Opin Drug Metab Toxicol. 2006;2:875-894.

20. Kobayashi K, Urashima K, Shimada N, Chiba K. Selectivities of human cytochrome P450 inhibitors toward rat P450 isoforms with cDNAexpressed systems of the rat. Drug Metab Dispos. 2003;31:833-836.

21. Nolin TD, Frye RF, Le P, et al. ESRD impairs nonrenal clearance of fexofenadine but not midazolam. J Am Soc Nephrol. 2009;20: 2269-2276.

22. Dowling TC, Briqlia AE, Fink JC, et al. Characterization of hepatic cytochrome p4503A activity in patients with end-stage renal disease. Clin Pharmacol Ther. 2003;73:427-434.
Clinical Pharmacology: Advances and Applications

\section{Publish your work in this journal}

Clinical Pharmacology: Advances and Applications is an international, peer-reviewed, open access journal publishing original research, reports, reviews and commentaries on all areas of drug experience in humans. The manuscript management system is completely online and includes a very quick and fair peer-review system, which is all easy to use.

\section{Dovepress}

Visit http://www.dovepress.com/testimonials.php to read real quotes from published authors. 